\title{
Exploratory behavior: The inadequacy of activity measures
}

M. H. SHELDON, DEPARTMENT OF PSYCHOLOGY, HULL UNIVERSITY, Hull, England

It has been reported that rats show lower activity and alternation scores in an elevated maze than they do in an enclosed maze, and it has been argued that this can be explained by a theory relating exploration to anxiety. But it is shown here that behavior in the two kinds of maze is so different that these comparisons do not justify the conclusions drawn from them.

Lester (1967a) has compared the locomotor activity of rats in an elevated and an enclosed $Y$ maze of similar dimensions and reported two differences. Rats in the elevated maze moved less often from one maze arm to another. They also showed a lower level of alternation between arms at the first opportunity: that is to say, having been placed in one arm (A), and having entered a second arm (B), they were less likely than rats in the enclosed maze to make their next entry into the third arm (C). Lester takes these results as supporting a theory of exploratory behavior (Lester, 1967b) according to which the probability of a rat's approaching a stimulus in its environment first increases and then decreases as a function of the fear aroused by that stimulus. The fear aroused by a stimulus is held to depend partly on its novelty, and partly on the rat's basal level of fear.

If this theory is to explain Lester's results, a number of assumptions have to be made. It must be assumed that rats in an elevated maze are generally more fearful than rats in an enclosed maze. Lester provides no independent evidence for this, but Halliday (1967) has recently reported that rats defecated more in an elevated maze. It must also be assumed that the measure Lester took of locomotor activity (the number of occasions on which a rat moved from one maze arm to another) is a measure of the rat's exploratory approaches to the stimuli in its environment, and further that it is an equally appropriate measure in both kinds of maze. But before we are justified in making this last assumption (and concluding, as Lester's theory predicts, that rats in elevated mazes explore less) we need to examine the behavior more carefully. An elevated and an enclosed maze may have identical ground plans, but they necessarily present the rat with very different sensory environments. In particular, of course, extramaze cues are likely to be far more prominent with an elevated maze. It may be, therefore, that there will be gross differences in behavior in the two situations, and that different patterns of response may have a claim to be called exploratory. If this proved to be so, comparisons based simply on Lester's measure of locomotor activity would obviously be unsatisfactory.
The following results (extracted from a longer experiment with rats in an elevated maze) seem to be relevant.

Method. The Ss were 18 hooded female rats, without previous maze experience, and about 100 days old. Each rat was observed for $3 \mathrm{~min}$ on each of three successive days in a wooden elevated $Y$ maze. The arms, measuring $18 \times 4$ in. were set at angles of $120 \mathrm{deg}$ to each other. The maze was supported 18 in. above the floor within an enclosure $4 \mathrm{ft} 6$ in. $x 4 \mathrm{ft} 6$ in. $x 4$ $\mathrm{ft}$ high, with walls of black muslin. A $100 \mathrm{~W}$ bulb hung 16 in. above the intersection of the arms; the testing room was in darkness.

By way of pretraining, rats were carried from the colony into the experimental room on each of the three days preceding the testing days, and placed on a table top where they were left for $5 \mathrm{~min}$. On testing days they were placed on the maze at the end of one of the arms, and facing away from the intersection. Various measures of behavior were taken, but the one reported here was the total time (to the nearest $1 / 2 \mathrm{sec}$ ) during a $3 \mathrm{~min}$ observation period for which the whole of a rat's head projected beyond the edge of the maze.

Results and Discussion. The results can be summarized very simply. On the three successive days of testing, rats spent an average of $61.5 \mathrm{sec}, 61.7 \mathrm{sec}$, and $60.7 \mathrm{sec}$ with their heads over the edge of the maze. Individual times ranged from $25 \mathrm{sec}$ to 98.5 sec. For much of this time their heads were below the level of the runways, and the rats gave every appearance of inspecting the extra-maze environment. During this time they were, of course, inactive in the sense that they were not moving from one arm of the maze to another.

In this experiment rats in an elevated maze spent on the average more than one third of their time engaging in behavior that was, by definition, impossible in an enclosed maze. Similar behavior has been noted (although not formally scored) by Halliday (1967). This behavior is incompatible with locomotor activity, and since it appears to be exploratory in the sense of being directed towards stimuli in the environment, it seems that to compare exploration in elevated and enclosed mazes simply by measuring activity is inappropriate.

In view of this evidence, similar objections apply to the conclusions Lester draws from the difference in initial alternation scores. It would be perfectly possible for rats to alternate with respect to cues outside the maze without also alternating between arms of the maze.

\section{References}

HALLIDAY, M. S., Exploratory behavior in elevated and enclosed mazes. Quart. J. exp. Psychol., 1967, 19, 254-263.

LESTER, D., Effects of fear upon exploratory behavior. Psychon. Sci, 1967a, 9, 117-118.

LESTER, D., Sex differences in exploration: Toward a theory of exploration. Psychol. Rec., 1967b, 17, 55-62. 